\title{
Amelioration of hyperlipidemia and coronary risk markers with supplementation of Cinnamomum zeylanicum bark extracts on Triton WR-1339 induced hyperlipidemia in Wistar albino rats
}

\author{
S. L. D. V. Ramana Murty Kadali ${ }^{1}$, B. H. M. R. K. Gupatha ${ }^{2, *}$, Vijay Kumar M. ${ }^{3}$, Revanasiddappa B. C. ${ }^{4}$ \\ ${ }^{1,2,3}$ Assistant Professor, ${ }^{4}$ Associate Professor, ${ }^{1,2}$ Dept. of Pharmacology, ${ }^{3,4}$ Dept. of Pharmaceutical Chemistry, \\ ${ }^{1}$ Modern Institute of Medical Sciences, Indore, Madhya Pradesh, ${ }^{2}$ IQ City Medical College, Durgapur, West Bengal, \\ ${ }^{3,4}$ NGSM Institute of Pharmaceutical Sciences, Mangalore, Karnataka, India \\ *Corresponding Author: \\ Email: manikanta05bayya@gmail.com
}

\begin{abstract}
Introduction: Cinnamomum zeylanicum bark used in Indian traditional medicine to treat hyperlipidemia and cardiovascular diseases. Hyperlipidemia is a major risk factor for coronary heart disease and metabolic syndrome.

Objective: The present study was to evaluate the antihyperlipidemic effect of ethanolic and aqueous extracts of Cinnamomum zeylanicum bark against Triton induced hyperlipidemia in Wistar albino rats.

Materials and Methods: Thirty rats (200-230gm) were randomly divided into five groups of six rats in each, received standard diet and water daily. Normal control (5\% CMC), positive control (Triton 400mg/kg), standard control (atorvastatin 40mg/kg), ethanolic and aqueous extracts of Cinnamon groups (CZEE and CZAE $500 \mathrm{mg} / \mathrm{kg}$ of each) were assigned and treated respectively. All the groups except normal control were administered triton to induce hyperlipidemia. Both the standard and plant extracts were administered for seven days orally. At the end of the study, fasting blood was collected and serum was separated out to estimate the lipid profile and various markers of coronary heart diseases.

Results: Post supplementation of atorvastatin, CZEE and CZAE significantly $(\mathrm{P}<0.05)$ ameliorated the hyperlipidemia and decreased the risk of cardiovascular diseases in triton induced hyperlipidemic rats.

Conclusion: From the observations, it can be concluded that supplementation of Cinnamon extracts can significantly decline the lipid abnormalities and ameliorate the markers of coronary heart disease and provides the scientific evidence for their traditional claims. Further molecular studies are warranted to evaluate the causative molecule for antihyperlipidemic effect.
\end{abstract}

Keywords: Hyperlipidemia, Cardiovascular diseases, Triton, Atorvastatin and Cinnamon extracts.

\section{Introduction}

Hyperlipidemia is a metabolic disorder characterized by elevated levels of total cholesterol (TC), low-density lipoprotein cholesterol (LDL-C), Very LDLC (VLDL-C) and triglycerides (TGs) with a subsequent decrease in the levels of high-density lipoprotein cholesterol (HDL-C) in the systemic circulation. ${ }^{1}$ Consumption of high fat or fructose diet, lifestyle modification, age, genetics, smoking, hypertension, type 2 diabetes and other precipitating factors play a significant role in causing aberrant lipid profile. Dyslipidemia is a major cause of atherosclerotic cardiovascular disease (ASCVDs), such as Coronary heart disease (CHD), ischemic cerebrovascular disease (CBVD) and peripheral vascular disease (PVD). ${ }^{2}$ Cardiovascular diseases (CVDs) are the major leading cause of worldwide morbidity and mortality among the adults. A 20\% reduction in blood cholesterol level can decrease about $31 \%$ of CHD incidence and $33 \%$ of its mortality rate. ${ }^{3}$

Hyperlipidemia is a primary target, to find a remedial measure for the treatment of CVD. Hypercholesteremia and hypertriglyceridemia are the potential risk factors either alone or together, responsible for the development of coronary artery disease and its progression towards atherosclerosis. ${ }^{4}$
Deposition of elevated LDL-C in the arterial endothelial spaces causes vascular toxicity and responsible for atherosclerosis, hypertension and obesity etc. In hyperlipidemia, both enzymatic as well as non-enzymatic antioxidative defense systems such as superoxide dismutase (SOD), lipid peroxidation (LPO), ascorbic acid and reduced glutathione (GSH) levels were reduced, leading to the generation of Reactive oxygen species (ROS) and which mediates vascular damage. $^{5}$

Currently available hypolipidemic agents like statins, fibrates, bile acid sequestrants and niacin were used either alone or in combinations to treat dyslipidemic disorders. On long-term use, they are well known to cause various side effects (includes hepatotoxicity, myopathy, hyperchloremic acidosis, dyspepsia, flushing, and pruritus), lacks safety, costeffectiveness and with poor patient's compliance. ${ }^{2}$ It indicates, there is a high demand in search of drugs with antihyperlipidemic, antioxidant activities with no or lesser side effects, and which can be accomplished by the phytomedicine. ${ }^{5-6}$ Recent literature reveals that the consumption of phyto-active constituents like polyphenols can abbreviate the risk of hyperlipidemia and associated complications. So, bioactive 
phytoconstituents will serve as leads for the design and development of effective, safe and economic drugs. ${ }^{6-7}$

Cinnamon bark is a spice, most commonly used as the aromatic and flavoring agent. Since ancient times, it is being used in traditional folkloric and ayurvedic medicine for treatment of several ailments. The bark is smooth, light to dark brown, with a strong, pleasant smell, spicy and a burning taste. There are two important varieties of cinnamon: Cinnamomum zeylanicum and Cinnamomum cassia. Cinnamomum zeylanicum (family- Lauraceae) is reported to have numerous pharmacological activities which includes anti-diabetic, ${ }^{8}$ hepatoprotective, ${ }^{9}$ anti-oxidant, ${ }^{10}$ antiinflammatory, ${ }^{11}$ anti-nociceptive, ${ }^{12}$ anti-fungal, ${ }^{13}$ antimicrobial, ${ }^{14}$ anti-viral, ${ }^{15}$ and anti-fertility. ${ }^{16}$ Literature unveils the lipid lowering effects of $C$. zeylanicum ${ }^{17-19}$ and C.cassia ${ }^{20}$ were carried out with different doses in diabetic (streptozotocin and alloxan) and hyperlipidemic (high fat or and fructose diet) models. Most of the research work accomplished on cinnamon species in hyperlipidemia due to an underlying disease of a multi-factorial origin. In the present study, aqueous and ethanolic extracts of $C$. zeylanicum with a tolerable dose were used, for a shorter duration in a particular hyperlipidemic model induced with Triton-WR 1339 and results were compared with atorvastatin.

\section{Material and Methods \\ Animals}

Wistar albino rats of either of sex, weighing 200$230 \mathrm{gm}$ were selected. All the animals were housed in polypropylene cages and a room temperature of $24 \pm 2^{\circ} \mathrm{C}$ and 12 hrs light and dark cycles were maintained throughout the period. The animals were allowed to acclimatize to the environment for seven days, prior to the experimentation. The ethical clearance was obtained from the Institutional animal ethics committee (IAEC), prior to the beginning of the experimentation with Ref. no. of NGSMIPS/IAEC/December-2016/36. Handling and care of laboratory animals were carried according to CPCSEA guidelines, Ministry of Forests \& Environment and Government of India.

\section{Drugs and Chemicals}

Triton WR-1339 and all other chemicals were of analytical grade, were procured from Sigma Aldrich, Himedia, and Loba chemie. The diagnostic kits are from Aspen diagnostics Ltd. Ethanolic and aqueous extracts of C. Zeylanicum bark (CIN/D26/STD02 and CIN/D26/STD01) were obtained from Greenchem, Bangalore, India.

\section{Triton-WR 1339 induced Hyperlipidemia ${ }^{21}$}

All the animals were randomly divided into five groups of six rats in each, fed with standard pellet diet and water daily for a period of seven days. The first group rats serve as normal control and received only $5 \%$ CMC (purified grade) orally. The other four groups were administered with a single intraperitoneal injection of a freshly prepared solution of Triton-WR $1339(400 \mathrm{mg} / \mathrm{kg})$, dissolved in normal saline to an overnight fasting (for $18 \mathrm{hr}$ ) rats. After 72 hours of triton injection, respective treatments were given once daily to all the animals. Second and third groups serve as a positive and standard control, received 5\% CMC and atorvastatin $(40 \mathrm{mg} / \mathrm{kg})$ respectively. Fourth and fifth group rats, were supplemented with ethanolic and aqueous extracts of Cinnamon bark (CZEE and CZAE $500 \mathrm{mg} / \mathrm{kg}$ of each) as shown in Table1. Both the standard and cinnamon extracts were suspended in 5\% CMC solution and were administered orally for seven days, after inducing hyperlipidemia.

Table 1: Animal grouping and treatment schedule in Triton induced hyperlipidemic rats respectively

\begin{tabular}{|c|c|}
\hline Groups $(n=6)$ & Treatment schedule \\
\hline I: Normal control & Received 5\% CMC solution orally \\
\hline II: Positive control & Received Triton $(400 \mathrm{mg} / \mathrm{kg}$, i.p) $+5 \%$ CMC solution orally \\
\hline III: Standard control & Received Triton + Atorvastatin (40mg/kg, p.o) \\
\hline IV: CZEE & Received Triton + Cinnamon ethanolic extract $(500 \mathrm{mg} / \mathrm{kg}, \mathrm{p} . \mathrm{o})$ \\
\hline $\mathrm{V}: \mathrm{CZAE}$ & Received Triton + Cinnamon aqueous extract (500mg/kg, p.o) \\
\hline \multicolumn{2}{|c|}{$\begin{array}{l}\text { Post-treatment for } 7 \text { days after induction of hyperlipidemia in albino rats. } \\
\text { CZEE-Cinnamon zeylanicum Ethanolic Extract, CZAE- Cinnamon zeylanicum } \\
\text { Aqueous Extract. }\end{array}$} \\
\hline
\end{tabular}

Most of the research works on plant extracts were carried out with a single daily dosing, ${ }^{22-23}$ may be to enhance the tolerability and to avoid multiple-dose cumulative toxicity ${ }^{24-25}$ of an unknown pharmacokinetic profile of a phytoconstituents, irrespective of screening of any pharmacological activities in animal models.

Collection of blood: On the eighth day, ${ }^{26} 2-3 \mathrm{ml}$ of fasting blood was collected by the retro-orbital puncture under mild ether anesthesia. Blood samples were centrifuged at 3000 RPM, with the help of ultra- centrifuge to separate the serum and stored at $-20^{\circ} \mathrm{C}$ until further biochemical analysis. ${ }^{27-28}$

Biochemical estimation: Serum total cholesterol, triglycerides were estimated by the method of CHODPAP and high-density lipoprotein-cholesterol by the method of GPO-PAP by using Semi-autoanalyzer and with the help of diagnostic kits.

VLDL-cholesterol level was determined by Friedewald's formula. ${ }^{29}$

VLDL-C $=\mathrm{TG} / 5$, 
LDL cholesterol was calculated as: ${ }^{29}$ LDL-C $=$ TC HDL-C- VLDL-C.

Determination of cardiac risk indices ${ }^{29-31}$

Atherogenic index and Percentage of protection were calculated by using the formula:

Atherogenic index (AI) $=(\mathrm{TC}-\mathrm{HDL}-\mathrm{C}) / \mathrm{HDL}-\mathrm{C}$.

$\%$ Protection $=$ AI of experimental control - AI of treated control / AI of control X 100.

Antiatherogenic index (AAI) = HDL-C X $100 /$ TCHDL-C.

Dyslipidemic marker or Coronary risk index (CRI) $=$ TC-C / HDL-C.

Cardiovascular risk index $($ CVRI $)=$ LDL-C $/$ HDLC.

Statistical Analysis: All the values were expressed as Mean \pm SEM and subjected to One-way analysis of variance followed by Tukey's multiple comparisons by using graph pad prism 5.0. $\mathrm{P}<0.05$ is considered as the level of significance.

\section{Results}

Effect on serum lipid Profile in hyperlipidemic rats Effect on serum level of TC

Positive control showed a significant increase in the levels of TC as compared to normal control rats. The rats treated with atorvastatin, CZEE and CZAE showed a significant decrease in the level of TC as compared to the positive control group after seven days of the respective treatments (Table 2). Both the CZEE and CZAE produce comparable effects with atorvastatin.

Table 2: The Effect of $C$. zeylanicum bark ethanolic and aqueous extracts on lipid profile in Triton induced hyperlipidemic rats

\begin{tabular}{|c|c|c|c|c|c|}
\hline Groups & $\begin{array}{c}\mathrm{TC} \\
\mathrm{mg} / \mathrm{dl}\end{array}$ & $\begin{array}{c}\text { TG } \\
\mathrm{mg} / \mathrm{dl}\end{array}$ & $\begin{array}{l}\text { HDL } \\
\mathrm{mg} / \mathrm{dl}\end{array}$ & $\begin{array}{l}\text { VLDL } \\
\mathrm{mg} / \mathrm{dl}\end{array}$ & $\begin{array}{c}\mathrm{LDL} \\
\mathrm{mg} / \mathrm{dl}\end{array}$ \\
\hline $\begin{array}{l}\text { Normal } \\
\text { control }\end{array}$ & $79.93 \pm 3.12^{\mathrm{b}, \mathrm{c}}$ & $145.6 \pm 7.37^{b}$ & $39.87 \pm 3.62$ & $29.11 \pm 1.47^{\mathrm{b}}$ & $10.95 \pm 3.67^{b, c}$ \\
\hline $\begin{array}{l}\text { Positive } \\
\text { control }\end{array}$ & $254.4 \pm 8.701^{\mathrm{a}, \mathrm{c}}$ & $434.8 \pm 65.6^{\mathrm{a}, \mathrm{c}}$ & $22.49 \pm 2.91^{\mathrm{c}}$ & $86.96 \pm 13.11^{\mathrm{a}, \mathrm{c}}$ & $144.9 \pm 9.85^{\mathrm{a}, \mathrm{c}}$ \\
\hline $\begin{array}{l}\text { Standard } \\
\text { control }\end{array}$ & $166.3 \pm 9.84^{\mathrm{a}, \mathrm{b}}$ & $167.8 \pm 8.41^{\mathrm{b}}$ & $51.87 \pm 3.23^{b}$ & $33.56 \pm 1.68^{b}$ & $80.87 \pm 10.99 \mathrm{a}, \mathrm{b}$ \\
\hline CZEE & $181.1 \pm 17.85^{\mathrm{a}, \mathrm{b}}$ & $182.1 \pm 10.60^{b}$ & $43.77 \pm 7.41^{\mathrm{b}}$ & $36.43 \pm 2.12^{b}$ & $100.9 \pm 26.47^{\mathrm{a}}$ \\
\hline CZAE & $204.5 \pm 6.11^{\mathrm{a}, \mathrm{b}}$ & $185.8 \pm 11.97^{\mathrm{b}}$ & $51.88 \pm 3.25^{\mathrm{b}}$ & $37.16 \pm 2.39^{b}$ & $115.4 \pm 7.28^{\mathrm{a}}$ \\
\hline
\end{tabular}

\section{Effect on serum level of TG's}

There is a significant increase in TG's levels in positive control in comparison with the normal control group. Whereas, the treatment with atorvastatin, CZEE and CZAE significantly reduced the TG's levels as compared to positive control (Table 2). A decrease of TG's levels with CZEE and CZAE were comparable with atorvastatin.

\section{Effect on serum level of HDL-C}

The positive control rats exhibited a significant decrease in the level of HDL-C, in comparison with normal control and also with the standard control group. The HDL-C levels in atorvastatin, CZEE and CZAE treatment groups were significantly increased in comparison with positive control group (Table 2). Both cinnamon extracts showed similar effects with atorvastatin in elevating the HDL-C in hyperlipidemic rats.

\section{Effect on serum level of VLDL-C}

Positive control exhibited a significant increase of VLDL-C levels, as compared to normal control groups. Whereas, administration of atorvastatin, CZEE and
CZAE, significantly decreased the levels of VLDL-C in comparison with positive control group. Treatment with CZEE and CZAE decreased VLDL-C in hyperlipidemic rats and effects were comparable with atorvastatin (Table 2).

\section{Effect on serum level of LDL-C}

LDL-C levels were significantly increased in positive control when compared with normal control rats. Treatment with atorvastatin exhibited a significant decrease in the positive control, whereas both cinnamon extracts fail to reduce LDL-C levels in hyperlipidemic rats (Table 2).

\section{Effect on AI, \% of protection and AAI}

AI is significantly elevated in positive control when compared with normal control. Treatment with atorvastatin and both cinnamon extracts significantly decreases the AI as compared to positive control. Moreover, positive control also exhibits a significant decrease of AAI in comparison with normal control rats. Similarly, in comparison with positive control, treatment with atorvastatin and both extracts of cinnamon, was potentially alleviated the AAI. The 
above results depict, that increase of AI and decrease of $\%$ of protection as well of AAI in the positive control, suggests the development of atherogenesis followed by the cardiovascular events in hyperlipidemic rats. Whereas, treatment with drugs could reverse the atherogenesis and offers protection from cardiovascular risks in hyperlipidemic rats (Fig. 1, 2 and 3).

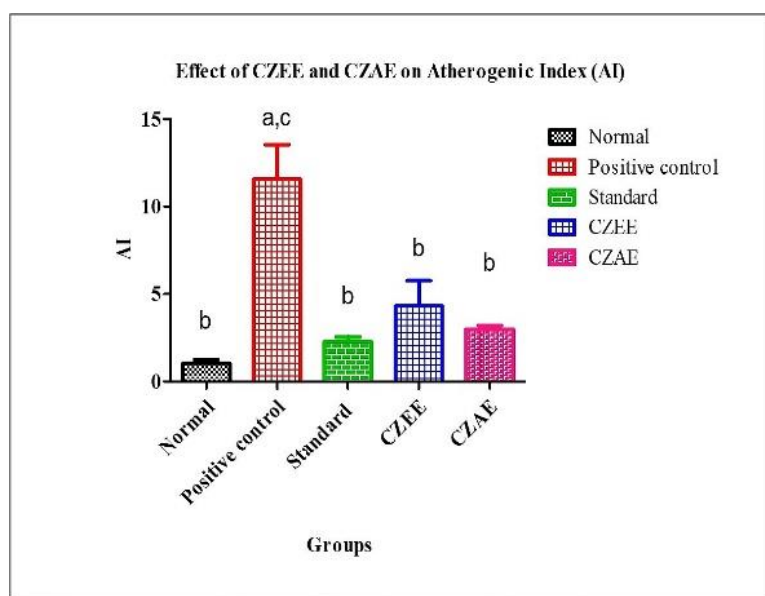

Fig. 1: The effect of CZEE and CZAE on AI in hyperlipidemic rats. Mean \pm SE $(n=6)$ a: Statistically significant from the nornmal control group; b: Statistically significant from the positive control group; c: Statistically significant from standard drug group

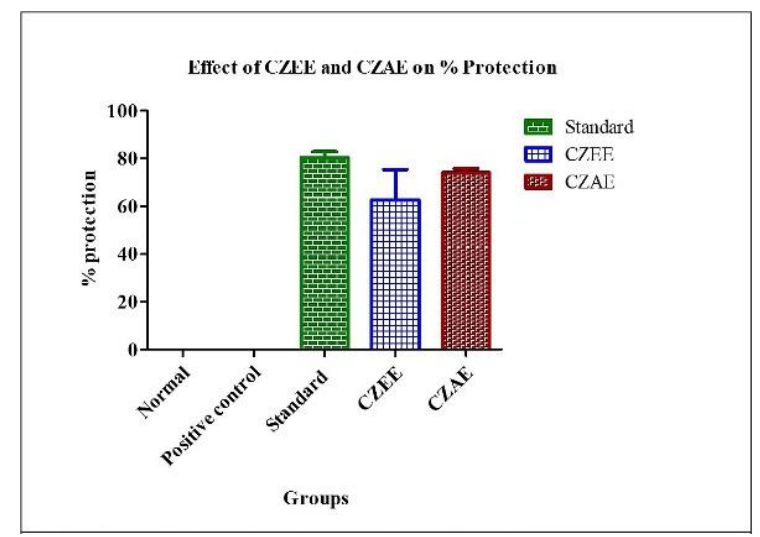

Fig. 2: The effect of CZEE and CZAE on \% protection in hyperlipidemic rats. Mean $\pm \mathrm{SE}(\mathrm{n}=6)$

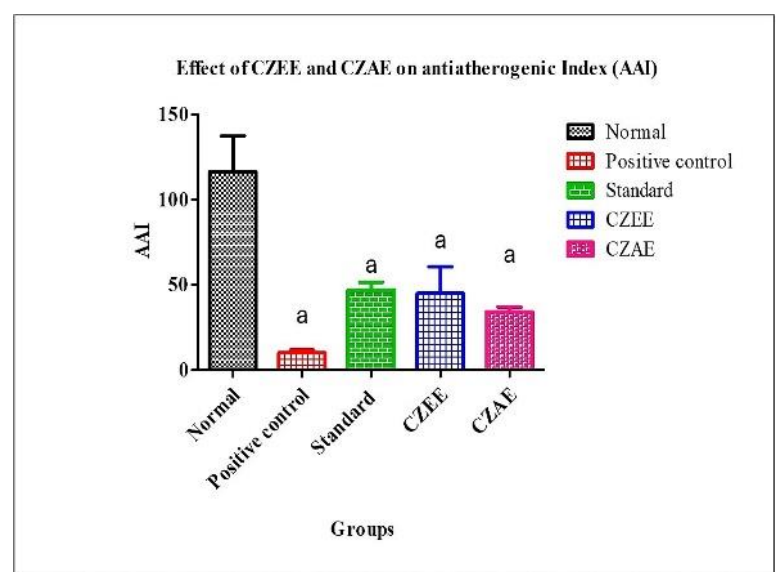

Fig. 3: The effect of CZEE and CZAE on AAI in hyperlipidemic rats. Mean \pm SE $(n=6)$ a: Statistically significant from the normal control group; b: Statistically significant from the positive control group; c: Statistically significant from standard drug group

Effect on a Dyslipidemic marker or Coronary risk index

The TC-C/HDL-C ratio is a marker of dyslipidemia, was significantly increased in the positive control, as compared to the normal control rats. Whereas, treatment with atorvastatin, CZEE and CZAE significantly decreased TC/HDL ratio in comparison to the positive control. Correction of dyslipidemia is the most significant result of treatment with $\mathrm{CZEE}, \mathrm{CZAE}$ and effects are comparable to atorvastatin. (Fig. 4)

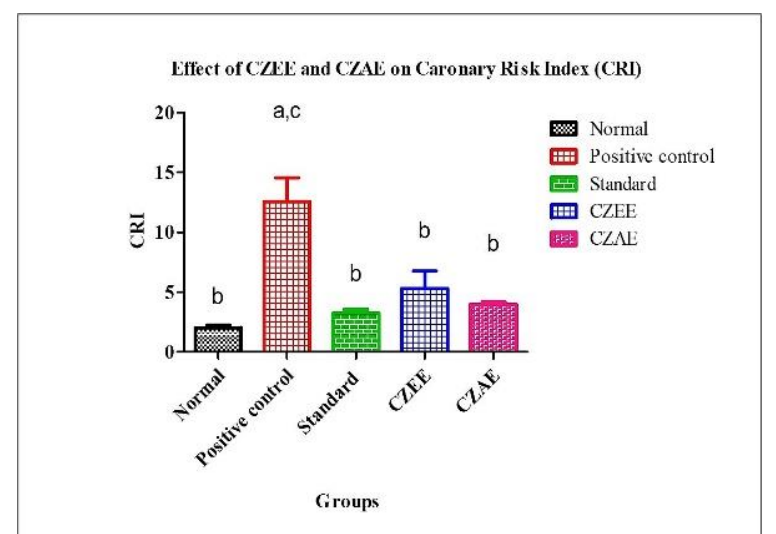

Fig. 4: The effect of CZEE and CZAE on CRI in hyperlipidemic rates. Mean \pm SE $(n=6)$. a: Statistically significant from the normal control group; b: Statistically significant from the positive control group; c: Statistically significant from the standard drug group

\section{Cardiovascular risk index (CVRI)}

CVRI is a ratio of LDL-C and HDL-C, was significantly higher in the positive control rats as compared to normal control rats. Treatment with atorvastatin, CZEE and CZAE significantly decreased CVRI in comparison with positive control rats. 
Atorvastatin profoundly decreased cardiovascular risk than the cinnamon extracts in hyperlipidemic rats. (Fig. $5)$.

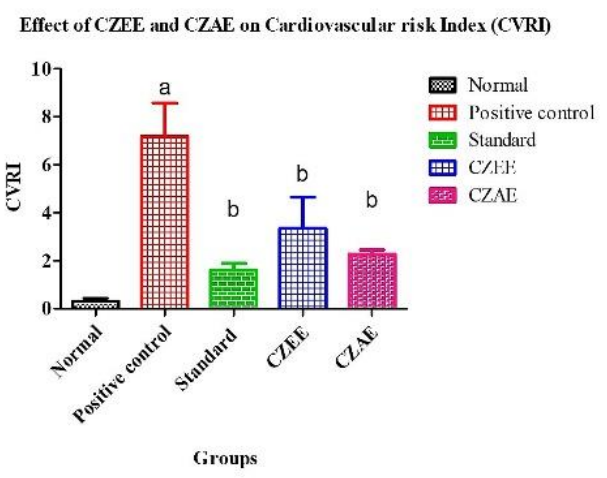

Fig. 5: The effect of CZEE and CZAE on CVRI in hyperlipidemic rats. Mean \pm SE $(n=6)$. a: Statistically significant from the normal control group; b: Statistically significant from the positive control group; c: Statistically significant from the standard drug group

\section{Discussion}

Hyperlipidemia is divided into primary and secondary subtypes. Primary hyperlipidemia is usually due to genetic (familial) causes and secondary (acquired) hyperlipidemia develops due to other underlying diseases. ${ }^{2}$ Normally the body balances the lipid metabolism between synthesis and degradation. When the balance is lost, lipid abnormalities can cause deleterious effects on arteries, blood pressure, body weight, insulin sensitivity, glucose utilization and fatty changes in the liver, etc. ${ }^{4}$ Increased levels of TC and lipoproteins (LDL-C and HDL-C) have a close correlation with the cardiovascular risks. TG's are composed of fatty acids and glycerol, circulate in the blood and stored in adipose tissue. In dyslipidemia, TG's get deposited in the cytoplasm of non-adipose cells, such as hepatocytes, muscle fibers and then closely linked to peripheral insulin resistance. ${ }^{21}$ When a high-fat diet is taken, TG's (and glucose) levels were significantly elevated and the body gradually processes the fat efficiently and their levels were decreased in a physiological manner. Approximately 65 percent of TC is carried by low-density lipoproteins. LDL-C (known as "bad" cholesterol) is potentially harmful, gets deposited onto the walls of arteries to undergo atherosclerosis. The particle size of cholesterol is utmost important because, larger particles are less dangerous than the smaller ones which readily penetrate the arterial wall and gets oxidized, which leads to the endothelial dysfunction, atheromatous plaque formation, ischemia and finally causes infraction. ${ }^{32}$ Atherogenesis is a process of oxidative modification of LDL, which triggers multiple pathological events leading to atherosclerosis. Clinically reported that
HDL-C (known as good cholesterol) is inversely related to TC and a decreased level of HDL-C can hasten the development of atherosclerosis leading to ischemic heart diseases, by impairing the cholesterol clearance from the arterial wall.

Now a days, many models are available, of which dietary manipulation or chemical induced hyperlipidemia is most commonly used animal models. Chronic consumption of high-fructose and or fat-rich diet-induced hyperlipidemias were associated with several manifestations of metabolic syndrome and type2 diabetes. Whereas, Triton WR-1339 or tyloxapol is a non-ionic detergent, widely used to induce acute hyperlipidemia in animals. The advantage of this model is the quick induction of hyperlipidemia, requires only a shorter treatment period and reproducibility, hence gained its popularity for the screening of natural or chemical entities for the hypolipidemic or antihyperlipidemic activity. ${ }^{27}$ Triton produces hypercholesterolemia and hypertriglyceridemia by accelerating hepatic cholesterol synthesis and blocking the clearance of TG-rich lipoproteins. ${ }^{28,33}$ Triton WR1339 acts as a surfactant and block the uptake of lipoproteins from the circulation through extra-hepatic pathways and increases the circulatory lipoprotein levels. Additionally, it increases the HMG-CoA reductase activity in the liver. ${ }^{34}$ The biphasic nature of triton-induced hyperlipidemia was helpful in understanding the mode of action of lipid-lowering agents. Drugs interfering with lipid biosynthesis or uptake will be active in the synthesis phase and metabolism will be active in the excretory phase. ${ }^{35-36}$ Moreover, the use of triton WR 1339 induced hyperlipidemia is an important approach to screen the action of hypolipidemic drugs but also as a mean for elucidating lipid metabolism. ${ }^{21}$

Research in recent years had been directed towards dietary supplements bearing antihyperlipidemic and antioxidants effects, derived from plant sources are beneficial in reducing atherogenic fractions and cardiovascular risks. ${ }^{37}$ There are a plethora of studies, which has investigated the medicinal plants for their acute hypolipidemic activity in Triton WR-1339induced hyperlipidemic animals.

In the present study, atorvastatin $(40 \mathrm{mg} / \mathrm{kg})$ is taken as standard drug, act as an HMG-CoA reductase inhibitor. It decreases hepatic cholesterol synthesis, increases LDL receptor expression on hepatocyte membranes and promotes LDL-C catabolism. By which, it reduces the TC, LDL-C and also the rate of production of VLDL-C by the liver. Hence, it's effective in treating patients with hypertriglyceridemia and hypercholesterolemia. ${ }^{2,4}$ Treatment with atorvastatin and both extracts of cinnamon exhibited a significant decrease in TC and improves HDL-C levels, indicates a significant improvement in the AI. AI indicates the deposition of atherosclerotic plaque or foam cells or fat infiltration in heart, coronaries, aorta, 
liver and kidneys results in oxidative damage to the organs. ${ }^{30}$ It correlates with the size of the pro-and antiatherogenic lipoprotein particles and is known to predict a CRI (TC-C/HDL-C ratio). ${ }^{29}$ AI value was higher in the positive control than normal control, is an indication of cardiovascular risk. Whereas, CRI was significantly reduced in treated groups with either atorvastatin, CZAE, and CZEE. Percentage of protection exhibited by all the treatment groups, confirms ameliorative effects of these drugs in the hyperlipidemia and associated cardiovascular risks in the present model. ${ }^{30}$

Antiatherogenic index (AAI) of positive control rats exhibited a bad impact of triton-induced dyslipidemia on the cardiovascular system. ${ }^{31}$ Supplementation of both extracts of cinnamon to hyperlipidemic rats, significantly alleviated the CVRI (LDL-C/HDL-C ratio), a marker of coronary heart diseases. ${ }^{31}$ The results, exhibit that reduction of these indices in treated hyperlipidemic rats strongly supported the belief that dietary supplementation with cinnamon may reduce the risk of developing cardiac diseases due to dyslipidemia.

The potential effects of Cinnamomum zeylanicum bark aqueous and ethanolic extracts on hyperlipidemia may be attributed to a decrease in the cholesterol synthesis and an increase in cholesterol excretion as well the expression of LDL receptors with subsequent metabolism and a parallel increase in HDL-C. The lipid-lowering effect of the selected model still there is some void existing to establish the same results to claim the qualitative results and a clear mechanistic approach is needed by conducting PK/PD profile which is planned to execute in the future.

\section{Conclusion}

Identifying the medicinal herbs effective in hyperlipidemia's is utmost important in terms of safety and cost-effective. Our findings suggest that cinnamon could be used as a dietary supplement in the treatment of hyperlipidemia, obesity and cardiac diseases. The antihyperlipidemic activity could be the due presence of aldehydes, chalcones, flavonoids, and polyphenols will claim the major credit in the protective mechanism. The precise mechanism of their biological effects vivo deserves further molecular investigation.

\section{Acknowledgments}

The authors are thankful to the co-workers of the Department and Management for facilitating to carry out this project expeditiously. We are also grateful to Greenchem, Bangalore for providing C. Zeylanicum extracts as gift sample.

Funding: No funding sources

Conflict of interest: None declared

\section{References}

1. Rohit G, Kim Kah H, Rajeev K. S, Ravi Chandra V, Sartaj Banu Mulapalli. "Antihyperlipidemic potential of Albiziaamara (Roxb) Boiv. bark against Triton X100 induced hyperlipidemic condition in rats" Pharmacognosy Res. 2014;6(4):267-273.

2. Holly E Gurgle, Donald K Blumenthal. Drug Therapy for Dyslipidemias. In: Laurence LB, Randa HD, Bjorn CK. editor's. Goodman and Gilman's. The Pharmacological Basis of Therapeutics. $13^{\text {th }}$ Ed. New York, McGraw-Hill; Chapter 33;2018;605-618.

3. Dhulasavant V, Shinde S, Pawar M, Naikwade NS. "Antihyperlipidemic activity of Cinnamomum tamala Nees. on high cholesterol diet induced". Int J PharmTech Res. 2010;2:2517-21.

4. Anil P, Nitin C, Tenpe CR, Yeole PG, Ravikiran P. "Effect of Atorvastatin and Hydroxychloroquine Combination on Triton WR 1339 Induced Hyperlipidemia in Rats". Inventi Rapid: Molecular Pharmacology,1(3).

5. Vijayaraj P, Muthukumar K, Sabarirajan J, Nachiappan V. "Antihyperlipidemic activity of Cassia auriculata flowers in Triton WR 1339 induced hyperlipidemic rats". ExpToxicolPathol. 2013;65(12):135-41.

6. Gayathri V, Ananthi S, Hannah R, Vasanthi. "Antihyperlipidemic potential of Polyphenol and Glycoside rich Nerium oleander flower against Triton WR-1339-induced hyperlipidemia in Experimental Sprague Dawley Rats". J Chem. 2013;01-08.

7. Kanika P Karmarkar SM, Bhagwat AM. "Evaluation of Antihyperlipidemic activity of Ficus Hispida Linn Leaves in Triton WR-1339 (Tyloxapol) induced hyperlipidemia in mice". Int J Pharm Pharm Sci. 2011;3(5):188-191.

8. Ranasinghe P, Perera S, Gunatilake M, Abeywardene E, Gunapala N, Premakumara S, et al. "Effects of Cinnamomum zeylanicum (Ceylon cinnamon) on blood glucose and lipids in a diabetic and healthy rat model." Phcog Res.2012;4:73-9.

9. Moselhy, SS Ali, HK. "Hepatoprotective effect of cinnamon extracts against carbon tetrachloride-induced oxidative stress and liver injury in rats". Biol.Res. 2009;42:93-98.

10. Singh G, Maurya S, Delampasona M.P. Catalan, C.A.N "A comparison of chemical, antioxidant and antimicrobial studies of cinnamon leaf and bark volatile oils, oleoresins and their constituents". Food and Chem Toxicol. 2007;45:1650-1661.

11. Badal R, Subhash B, Mohan V, Prasad T. "Ameliorative Effects of a Polyphenolic Fraction of Cinnamomum zeylanicum L. Bark in animal models of Inflammation and Arthritis". Sci Pharm. 2013;81:567-589.

12. Atta AH, Alkofahi A. "Anti-nociceptive and antiinflammatory effects of some Jordanian medicinal plant extracts". J Ethnopharmacol. 1998.60:117-124.

13. Ana Carolina P. M, Edeltrudes de O. L, Evandro L. de S, Monique Araujo V. D, Vinícius N. T. "Inhibitory Effect of Cinnamomum Zeylanicum Blume (Lauraceae) Essential oil and $\beta$-Pinene on the growth of dematiaceous moulds". Brazilian Journal of Microbiology.2007;38:3338.

14. Mehmet Unlu, Emel Ergene, Gulhan Vardar U, Hulya Sivas Z, NiluferVural. "Composition, antimicrobial activity and in vitro cytotoxicity of essential oil from Cinnamomum zeylanicum Blume (Lauraceae)". Food and Chemical Toxicology.2010;48:3274-3280.

15. Tamam S Ma, Abd el Hamid M Sb, Samah M Helmyc, Marwa A Nakhriry. "The Anti-viral and Immunomodulatory activity of Cinnamon zeylanicum 
against Newcastle disease virus in chickens". IJSBAR. 2017;32(2):251-262.

16. Dalia A, Hafez. "Effect of Extracts of Ginger goots and Cinnamon bark on fertility of male diabetic rats". Journal of American Science. 2010;6(10):940-947.

17. Rekha. N, Balaji. R, Deecaraman M Antihyperglycemic and antihyperlipidemic effects of extracts of the pulp of Syzygium cumini and bark of Cinnamon zeylanicum in streptozotocin-induced diabetic rats. J. Appl. Biosci. 2010. 28:1718-1730.

18. Kannappan S, Jayaraman T, Rajasekar R, Ravichandran MK, Anuradha CV. "Cinnamon bark extract improves glucose metabolism and lipid profile in the fructose-fed rat". Singapore Med J. 2006;47:858-863.

19. Zeynep T, Cemal O, Nurhan S, Vijaya J, Kazim S. "Cinnamon Polyphenol extract inhibits hyperlipidemia and inflammation by modulation of transcription factors in high-fat diet-fed rats". Oxidative Medicine and Cellular Longevity.2017;1-10.

20. Husni Abdulla MD, Amad M. Saleh J, Malika Kassim N. Impact of Cinnamon Extract on Hyperlipidemic and Diabetic Rats. International Journal of Chemical and Biomolecular Science. 2015; (3):96-106.

21. Girija K, Lakshman K Anti-hyperlipidemic activity of methanol extracts of three plants of Amaranthus in tritonWR 1339 induced hyperlipidemic rats Asian Pacific J Trop Biomed 2011;62-65.

22. Syed Safiullah G, Rizwan Khan M.A, Kaab EA, Abid Hussain A. Evaluation of antihyperlipidemic activity of ethanolic extract of Glycosmis pentaphylla in hyperlipidemic wistar rats. International Journal of Pharma Sciences and Research. 2015;6(2):282-292.

23. Uchendu I.K, Onwukwe OS, Chidozie EA, Oliver C Orji, Blessing E C. Anti-hyperlipidemic effect of crude methanolic extracts of Glycine max (soy bean) on high cholesterol diet-fed albino rats. J Med Allied Sci. 2017;7(1):34-40

24. Tayyaba A, Suhail R, Rashid Khan.MD, Almajwal A. Anti-depressant and anxiolytic potential of Acacia hydaspica R. Parker aerial parts extract: Modulation of brain antioxidant enzyme status. BMC Complementary and Alternative Medicine.2017;17:228-240.

25. Abrar HM, Manjusha S, Yousuf Malla MD. An acute oral toxicity study of methanolic extract from Tridex procumbens in Sprague Dawley's Rats as per OECD guidelines 423. Asian Journal of Plant Science and Research, 2013,3(1):16-20.

26. Shobana G, Sivaelango G, Kumaravel P, Evaluation of anti-hyperlipidaemic effect of Macrotyloma uniflorum seed extract in experimental hyperlipidaemia.2012;3(5):335-356.

27. Sundarrajan T, Raj kumar T, Udhaya kumar E, Arunachalam G. "Hypolipidemic activity of Pithecellobium dulce Bench. in Triton Wr-1339 induced hyperlipidemic rats". International Journal of Chemical and Pharmaceutical Sciences. 2010;1(2):50-53.

28. Samrat AK, Pratibha BA. "Synthesis, characterization and antihyperlipidemic activity of novel condensed pyrazole [3,4-d] pyrimidine derivatives". Der Pharma Chemica.2014;6(4):214-222.

29. Yogendra N, Venkatachalam H, Vijay Kumar D, Geetha M, Jayashree BS, Unnikrishnan MK. Antidiabetic activity of 3-Hydroxyflavone analogues in high fructose-fed insulin resistant rats". EXCLI Journal.2014;13:10551074.

30. Munshi RP, Joshi SG, Rane BN. "Development of an experimental diet model in rats to study hyperlipidemia and insulin resistance, markers for coronary heart disease". Indian J Pharmacol.2014;46:270-6.

31. Mahmoud AM, Hozayen WG, Soliman HA, Mostafa SR. "Enteromorphaflexuosa improves insulin sensitivity and metabolic control in fructose-induced diabetic rats". $J$ Endocrinol Diabetes Obes.2015;3(2):1072.

32. Gajendra K, Amita S, Surinder KS, Yogendra KG. "The hypolipidemic activity of Ayurvedic medicine, Arogyavardhinivati in Triton WR-1339-induced hyperlipidemic rats: A comparison with fenofibrate". J Ayur Integrative Med. 2013;4(3):165-170.

33. Nandakumar K, Singh R, Bansal SK, Bodhankar SL, Jindal D. Coumar MS, Bhardwaj SH. "Effect of curcumin on Triton WR 1339 induced hypercholesterolemia in mice". Indian J Pharmacol. 2004;36(6):381-384.

34. Guptha BHMRK, Ramana Murty Kadali SLDV, Vijay Kumar M., Revanasiddappa BC. "Antihyperlipidemic activity of Chloroxylon swietenia in Triton WR1339 induced hyperlipidemia”. Int J Basic Clin Pharmacol. 2018;7(3):518-523.

35. Kripi V, Vivek KG, Harish D, Vandana G. "Antihyperlipidemic Activity of Lens culinaris medikus seeds in Triton WR1339 induced hyperlipidemic rats". $J$ Pharmacogn Nat Prod.,2016;2(2):2-7.

36. Ananthi T, Jasmin B, Chitra M. "Antihyperlipidemic activity of Micheliachampaca L. in Triton WR 1339 induced albino Rats". Int J PharmTech Res.2014;6(4):1368-373.

37. Pankaj GJ, Sanjay JS. "A Review of Indian Medicinal Plants with hypolipidemic activity and their medicinal importance world". J Pharma Pharm Sci.2015;4(3):14771493. 